\title{
Endo-Noetherian rings
}

\author{
Badreddine Gouaid $^{1}$. Ahmed Hamed ${ }^{1}$. Ali Benhissi ${ }^{1}$
}

Received: 29 January 2019 / Accepted: 9 July 2019 / Published online: 22 July 2019

(c) Fondazione Annali di Matematica Pura ed Applicata and Springer-Verlag GmbH Germany, part of Springer Nature 2019

\begin{abstract}
A commutative ring $R$ is said to be endo-Noetherian if the chain of annihilators ann $\left(a_{1}\right) \subseteq$ $\operatorname{ann}\left(a_{2}\right) \subseteq \cdots$ stabilizes for each sequence $\left(a_{k}\right)_{k}$ of elements of $R$ (Ndiaye and Gueye in Int J Appl Math 86:871-881, 2013). In this paper, we give equivalent conditions for the power series (resp., polynomial) rings over an Armendariz ring to be endo-Noetherian. We also study several properties of an endo-Noetherian ring. The concept of the amalgamated duplication of $R$ along an ideal $I, R \bowtie I$ is studied.
\end{abstract}

Keywords Endo-Noetherian rings · Armendariz rings · Power serieswise Armendariz rings

Mathematics Subject Classification 13E99 1 13F25 $\cdot$ 13F20

\section{Introduction}

All the rings considered are commutative with identity. An $R$-module $M$ is called endoNoetherian if it satisfies the ascending chain condition on endomorphic kernels, i.e. the chain $\operatorname{Ker}\left(f_{1}\right) \subseteq \operatorname{Ker}\left(f_{2}\right) \subseteq \cdots$ stabilizes for each sequence $\left(f_{k}\right)_{k}$ of endomorphisms of $M$ [17]. A commutative ring $R$ is said to be endo-Noetherian if it is endo-Noetherian as an $R$-module. Equivalently, $R$ is endo-Noetherian if the chain of annihilators $\operatorname{ann}\left(a_{1}\right) \subseteq \operatorname{ann}\left(a_{2}\right) \subseteq \cdots$ stabilizes for each sequence $\left(a_{k}\right)_{k}$ of elements of $R$. The class of endo-Noetherian rings includes Noetherian rings and iso-Noetherian rings (Recall from [7] that an $R$-module $M$ is iso-Noetherian if for every ascending chain $M_{1} \subseteq M_{2} \subseteq \cdots$ of submodules of $M$, there exists an index $n \geq 1$ such that $M_{n} \simeq M_{i}$ for every $i \geq n$. We say that a ring $R$ is iso-Noetherian if the $R$-module $R$ is iso-Noetherian). The purpose of this paper is to characterize when the power series (resp., polynomial) rings over Armendariz rings are endo-Noetherian.

\footnotetext{
$\bowtie \quad$ Ahmed Hamed

hamed.ahmed@hotmail.fr

Badreddine Gouaid

bgouaid@gmail.com

Ali Benhissi

ali_benhissi@yahoo.fr

1 Department of Mathematics, Faculty of Sciences, Monastir, Tunisia
} 
In the first part of this paper, we study several properties of an endo-Noetherian ring. We show that if $R$ is a commutative ring and $S$ a multiplicative subset of $R$ consisting of regular elements, then $R$ is endo-Noetherian if and only if $S^{-1} R$ is endo-Noetherian. Also, we construct an example of a commutative ring $R$ and a multiplicative subset $S$ of $R$ such that $R_{S}$ is Noetherian (so endo-Noetherian) but $R$ is not endo-Noetherian. We study when the direct product of rings is endo-Noetherian. We prove that if $\left(R_{i}\right)_{i \in I}$ is a family of commutative rings and $R=\prod_{i \in I} R_{i}$ the product of $R_{i}$, then $R$ is endo-Noetherian if and only if $I$ is a finite set and for each $i \in I, R_{i}$ is endo-Noetherian. Let (*) be the following property: every increasing sequence of the form $\left(I: a_{1}\right) \subseteq\left(I: a_{2}\right) \subseteq \cdots$ is stationary, where $I$ is an ideal of $R$ and $\left(a_{k}\right)_{k}$ a sequence of elements of $R$. Note that if $R$ satisfies the property (*), then $R$ satisfies the accr condition (we say that $R$ satisfies the accr condition if the ascending chain of colon ideals of the form $(I: a) \subseteq\left(I: a^{2}\right) \subseteq\left(I: a^{3}\right) \subseteq \cdots$ is stationary for each $a \in R$ and every ideal $I$ of $R$ ). We show that $R$ satisfies the property (*) if and only if $R / I$ is an endo-Noetherian ring for each ideal $I$ of $R$. We also study the amalgamated duplication of $R$ along an ideal $I, R \bowtie I$ to be endo-Noetherian. The amalgamated duplication of a ring $R$ along an ideal $I$ is a ring that is defined as the following subring with unit element $(1,1)$ of $R \times R$ :

$$
R \bowtie I=\{(r, r+i) \mid r \in R, i \in I\} .
$$

This construction has been studied, in the general case, and from the different points of view of pullbacks, by D'Anna and Fontana [5]. According to [6], an element of $R$ is called a regular element if it is not a zerodivisor. Also, we say that $I$ is a regular ideal if it contains a regular element of $R$. We prove that if $I$ is a regular ideal of $R$, then $R$ is endo-Noetherian if and only if $R \bowtie I$ is endo-Noetherian. In the second part of this paper, we give equivalent conditions for the power series (resp., polynomial) rings over an Armendariz ring to be endoNoetherian. First, we need to collect some necessary notions. Recall that a ring $R$ is called an Armendariz ring if whenever the polynomials $f=\sum_{i=0}^{m} a_{i} X^{i}$ and $g=\sum_{i=0}^{n} b_{i} X^{i} \in R[X]$ satisfy $f g=0$ we have $a_{i} b_{j}=0$ for every $i$ and $j$ [14]. Also, $R$ is said power serieswise Armendariz if for each $f=\sum_{i \geq 0} a_{i} X^{i}$ and $g=\sum_{i \geq 0} b_{i} X^{i} \in R \llbracket X \rrbracket$ such that $f g=0$, then $a_{i} b_{j}=0$ for each $i$ and $j$. We prove that if $R$ is an Armendariz commutative ring, then the following are equivalent:

1. $R[X]$ is endo-Noetherian.

2. $R$ satisfies the acc on annihilators of finite subset.

3. $R$ satisfies the acc on annihilators of finitely generated ideals of $R$.

Finally, if $R$ is a power serieswise Armendariz ring, then the following statements are equivalent:

1. $R \llbracket X \rrbracket$ is endo-Noetherian.

2. $R$ satisfies the acc on annihilators of countably subset.

3. $R$ satisfies the acc on annihilators of countably generated ideals of $R$.

4. For each sequence $\left(f_{k}=\sum_{j \geq 0} a_{k, j} X^{j}\right)_{k}$ of elements of $R \llbracket X \rrbracket$ such that $a n n_{R \llbracket X \rrbracket}\left(f_{1}\right) \subseteq$ $a_{R \| X \rrbracket}\left(f_{2}\right) \subseteq \cdots$, there exists an $n \in \mathbb{N}$ such that for each $k \geq n, \cap_{j \geq 0} a n_{R}\left(a_{k, j}\right)=$ $\cap_{j \geq 0} \operatorname{ann}_{R}\left(a_{n, j}\right)$. 


\section{General properties}

Let $R$ be a commutative ring. According to [17], the ring $R$ is said to be endo-Noetherian if the increasing sequence $\operatorname{ann}\left(a_{1}\right) \subseteq \operatorname{ann}\left(a_{2}\right) \subseteq \cdots$ stabilizes for any sequence $\left(a_{k}\right)_{k}$ of elements of $R$. In this section, we study several properties of the endo-Noetherian ring.

Example 2.1 1. Every Noetherian ring is an endo-Noetherian ring.

2. The converse of (1) is not true in general. Let $R$ be a commutative ring with identity and $M$ an $R$-module. The idealization of $M$ in $R$ (or trivial extension of $R$ by $M$ ) is a commutative ring $R(+) M=\{(r, m) \mid r \in R$ and $m \in M\}$ under the usual addition and the multiplication defined as $\left(r_{1}, m_{1}\right)\left(r_{2}, m_{2}\right)=\left(r_{1} r_{2}, r_{1} m_{2}+r_{2} m_{1}\right)$ for all $\left(r_{1}, m_{1}\right),\left(r_{2}, m_{2}\right) \in R(+) M$. Now, let $K$ be a field and $V$ a $K$-vector space, then $K(+) V$ is an endo-Noetherian ring, because for each $(k, m) \in K \times V \backslash(0,0)$,

$$
\operatorname{ann}_{K(+) V}((k, m))= \begin{cases}(0,0) & \text { if } k \neq 0 \\ 0(+) V & \text { if } k=0\end{cases}
$$

Note that if $V$ has an infinite dimensional, then by [2, Theorem 4.8], $K(+) V$ is not Noetherian.

3. Recall from [7] that an $R$-module $M$ is iso-Noetherian if for every ascending chain $M_{1} \subseteq M_{2} \subseteq \cdots$ of submodules of $M$, there exists an index $n \geq 1$ such that $M_{n} \simeq M_{i}$ for every $i \geq n$. We say that a ring $R$ is iso-Noetherian if the $R$-module $R$ is iso-Noetherian. By [7, Lemma 5.4(1)], every iso-Noetherian ring is endo-Noetherian.

Recall that a commutative ring $R$ is said to be strongly Hopfian if the chain of annihilators $\operatorname{ann}(a) \subseteq \operatorname{ann}\left(a^{2}\right) \subseteq \cdots$ is stationary for each $a \in R[3,9,12,13]$. Note that every endoNoetherian ring satisfies the strongly Hopfian property. In Example 2.7, we provide a strongly Hopfian ring which is not endo-Noetherian.

Proposition 2.2 Let $R$ be a commutative ring and $S$ a multiplicative subset of $R$ consists of regular elements. Then, the following assertions are equivalent:

1. $R$ is endo-Noetherian.

2. $S^{-1} R$ is endo-Noetherian.

Proof $(1) \Rightarrow(2)$. Let $\left(\frac{a_{k}}{s_{k}}\right)_{k}$ be a sequence of elements of $S^{-1} R$ such that $a^{n n n_{S^{-1}}}\left(\frac{a_{1}}{s_{1}}\right) \subseteq$ $\operatorname{ann}_{S^{-1} A}\left(\frac{a_{2}}{s_{2}}\right) \subseteq \cdots$. We will show that $\operatorname{ann}_{R}\left(a_{k}\right) \subseteq \operatorname{ann}_{R}\left(a_{k+1}\right)$ for each $k \in \mathbb{N}$. Let $b \in$ $\operatorname{ann}_{R}\left(a_{k}\right)$. Since $\frac{b}{1} \in a n n_{S^{-1} R}\left(\frac{a_{k}}{s_{k}}\right), \frac{b}{1} \in a n n_{S^{-1} R}\left(\frac{a_{k+1}}{s_{k+1}}\right)$. This implies that $b \in \operatorname{ann}_{R}\left(a_{k+1}\right)$, because $S$ consists of regular elements.

Now, as $R$ is endo-Noetherian, there exists a positive integer $n$ such that for each $k \geq n$, $\operatorname{ann}\left(a_{k}\right)=\operatorname{ann}\left(a_{n}\right)$. Let $k \geq n$ and let $\frac{b}{t} \in \operatorname{ann}\left(\frac{a_{k}}{s_{k}}\right)$. Then, $b a_{k}=0$; so $b a_{n}=0$ which implies that $\frac{b}{t} \in \operatorname{ann}\left(\frac{a_{n}}{s_{n}}\right)$. Thus, the chain $a n n_{S^{-1} R}\left(\frac{a_{1}}{s_{1}}\right) \subseteq \operatorname{ann}_{S^{-1} R}\left(\frac{a_{2}}{s_{2}}\right) \subseteq \cdots$ is stationary, and hence the ring $S^{-1} R$ satisfies the endo-Noetherian property.

(2) $\Rightarrow(1)$. Assume that $S^{-1} R$ is endo-Noetherian and let $\left(a_{k}\right)_{k}$ be a sequence of elements of $R$ such that $\operatorname{ann}\left(a_{1}\right) \subseteq \operatorname{ann}\left(a_{2}\right) \subseteq \cdots$. Since $S$ consists of regular elements, for each $k \in \mathbb{N}^{*}, a^{a n n_{S^{-1}}}\left(\frac{a_{k}}{1}\right) \subseteq a n n_{S^{-1} R}\left(\frac{a_{k+1}}{1}\right)$. Thus by hypothesis, there exists a nonzero positive integer $n$ such that for each $k \geq n$, ann $S^{-1} R\left(\frac{a_{n}}{1}\right)=a n n_{S^{-1} R}\left(\frac{a_{k}}{1}\right)$. Now, let $k \geq n$, and $\alpha \in \operatorname{ann}\left(a_{k}\right)$, then $\frac{\alpha}{1} \in a n n_{S^{-1} R}\left(\frac{a_{k}}{1}\right)=a n n_{S^{-1} R}\left(\frac{a_{n}}{1}\right)$; so $\alpha a_{n}=0$, because $S$ consists of regular elements. This implies that $\operatorname{ann}\left(a_{n}\right)=\operatorname{ann}\left(a_{k}\right)$ for each $k \geq n$. Hence, $R$ is endo-Noetherian. 
We next construct an example of a commutative ring $R$ and a multiplicative subset $S$ of $R$ such that $R_{S}$ is Noetherian (so endo-Noetherian) but $R$ is not endo-Noetherian. To prove this, we need to collect some necessary notions. Let $R$ be a commutative ring and $S$ be a multiplicative subset of $R$. Recall from [1] (or [10]) that an ideal $I$ of $R$ is called $S$-finite (resp., $S$-principal), if $s I \subseteq J \subseteq I$ for some finitely generated (resp., principal) ideal $J$ of $R$ and some $s \in S$. Also, $R$ is called $S$-Noetherian if each ideal of $R$ is $S$-finite. In [1], the authors showed that $R$ is $S$-Noetherian if and only if every prime ideal is $S$-finite.

Example 2.3 Let $F$ be a field, $\mathbf{X}=\left\{X_{i} \mid i \in \mathbb{N}\right\}$ a set of indeterminates over $F, I$ the ideal of $F[\mathbf{X}]$ generated by the set $\left\{X_{0}^{j} X_{j} \mid j \geq 1\right\}$ and $R=F[\mathbf{X}] / I$. For each $i \in \mathbb{N}$, let $\overline{X_{i}}$ denote the homomorphic image of $X_{i}$ in $R$. Let $S=\left\{{\overline{X_{0}}}^{i} \mid i \in \mathbb{N}\right\}$ and $\overline{\mathbf{X}}=\left\{\overline{X_{i}} \mid i \in \mathbb{N}\right\}$. Then, $S$ is a multiplicative subset of $R$.

1. $R$ is not strongly Hopfian. Indeed, let $a=\overline{X_{0}}$, then for each $n \in \mathbb{N}^{*}, \overline{X_{n}} \in$ $\operatorname{ann}\left(a^{n}\right) \backslash \operatorname{ann}\left(a^{n-1}\right)$. So $R$ is not endo-Noetherian.

2. Let $P$ be the prime ideal of $R$ generated by the set $\{\overline{\mathbf{X}}\}$. Then, $P$ is the only prime ideal of $R$. Since $\overline{X_{0}} \in P, \overline{X_{0}} P \subseteq\left(\overline{X_{0}}\right) \subseteq P$; so $P$ is an $S$-finite ideal of $R$. This implies that $R$ is an $S$-Noetherian ring, and hence $R_{S}$ is Noetherian [1, Proposition 2(f)]. In particular, $R$ is an $S$-Noetherian ring which is not Noetherian.

Let $R$ be a commutative ring and $S$ a multiplicative subset of $R$. Our next result gives a sufficient condition for the localization of $R, R_{S}$ satisfies the endo-Noetherian property implies that $R$ is endo-Noetherian.

Proposition 2.4 Let $R$ be a quasi-local ring with maximal ideals $M_{1}, \ldots, M_{n}$. If $\left(R \backslash M_{i}\right)^{-1} R$ is an endo-Noetherian ring for each $1 \leq i \leq n$, then $R$ is endo-Noetherian.

Proof Let $\left(a_{k}\right)_{k}$ be a sequence of elements of $R$ such that $\operatorname{ann}_{R}\left(a_{1}\right) \subseteq \operatorname{ann}_{R}\left(a_{2}\right) \subseteq \cdots$. It is easy to show that $a n n_{\left(R \backslash M_{i}\right)^{-1} R}\left(\frac{a_{1}}{1}\right) \subseteq \operatorname{ann}_{\left(R \backslash M_{i}\right)^{-1} R}\left(\frac{a_{2}}{1}\right) \subseteq \cdots$ for each $1 \leq i \leq n$. Note that for each $1 \leq i \leq n$, there exists a positive integer $m_{i}$ such that for each $k \geq$ $m_{i}$, $\operatorname{ann}_{\left(R \backslash M_{i}\right)^{-1} R}\left(\frac{a_{k}}{1}\right)=\operatorname{ann}_{\left(R \backslash M_{i}\right)^{-1} R}\left(\frac{a_{m_{i}}}{1}\right)$. Let $m=\max \left\{m_{i} \mid 1 \leq i \leq n\right\}$. Then, for each $1 \leq i \leq n$ and each $k \geq m$, $\operatorname{ann}_{\left(R \backslash M_{i}\right)^{-1} R}\left(\frac{a_{k}}{1}\right)=a n n_{\left(R \backslash M_{i}\right)^{-1} R}\left(\frac{a_{m}}{1}\right)$.

Now, we will prove that for each $k \geq m$, $\operatorname{ann}_{R}\left(a_{k}\right)=a n_{R}\left(a_{m}\right)$. Let $k \geq m$ and $b \in$ $\operatorname{ann}_{R}\left(a_{k}\right)$. Then $b a_{k}=0$, and for each $1 \leq i \leq n, \frac{b}{1} \frac{a_{k}}{1}=0$ in $\left(R \backslash M_{i}\right)^{-1} R$; so for each $1 \leq i \leq n, \frac{b}{1} \frac{a_{m}}{1}=0$. This implies that for each $1 \leq i \leq n$, there exists an $s_{i} \in R \backslash M_{i}$ such that $s_{i} b a_{m}=0$. As the ideal generated by $\left\{s_{1}, \ldots, s_{n}\right\}$ is equal to $R$, we deduce that $b a_{m}=0$, and hence $R$ is endo-Noetherian.

Theorem 2.5 Let $\left(R_{i}\right)_{i \in I}$ be a family of commutative rings and $R=\prod_{i \in I} R_{i}$ the product of $R_{i}$. Then, $R$ is endo-Noetherian if and only if $I$ is finite and for each $i \in I, R_{i}$ is endoNoetherian.

Proof The "only if" part assume that $R=\prod_{i \in I} R_{i}$ is endo-Noetherian. If $I$ is not finite, then the sequence $\operatorname{ann}_{R}((0,1,1, \ldots)) \subseteq \operatorname{ann}_{R}((0,0,1,1, \ldots)) \subseteq \cdots$ does not stabilize. It is easy to prove that if $R=\prod_{i=1}^{n} R_{i}$ is endo-Noetherian, then for each $1 \leq i \leq n, R_{i}$ is endo-Noetherian.

For "if" part, let $R_{1}, \ldots, R_{n}$ be endo-Noetherian rings. We will show that $R=\prod_{i=1}^{n} R_{i}$ is endo-Noetherian. To prove this, it sufficient to show the proof in the case $n=2$ and conclude by induction on $n$.

Let $R=A \times B$ and $\left(a_{1}, b_{1}\right), \ldots \in R$ such that $\operatorname{ann}_{R}\left(\left(a_{1}, b_{1}\right)\right) \subseteq \cdots$. It is easy to show that $\operatorname{ann}_{A}\left(a_{1}\right) \subseteq \operatorname{ann}_{A}\left(a_{2}\right) \subseteq \cdots$ and $\operatorname{ann}_{B}\left(b_{1}\right) \subseteq \operatorname{ann}_{B}\left(b_{2}\right) \cdots$. Since $A$ and $B$ are 
endo-Noetherian rings, there exists a positive integer $n$ such that for each $k \geq n, \operatorname{ann}_{A}\left(a_{k}\right)=$ $\operatorname{ann}_{A}\left(a_{n}\right)$ and $\operatorname{ann}_{B}\left(b_{k}\right)=a n_{B}\left(b_{n}\right)$. This implies that for each $k \geq n$, ann $R\left(\left(a_{k}, b_{k}\right)\right)=$ $\operatorname{ann}_{R}\left(\left(a_{n}, b_{n}\right)\right)$, and hence $R$ is endo-Noetherian.

Corollary 2.6 Let $\left(R_{i}\right)_{1 \leq i \leq n}$ be a finite family of commutative rings and $R=\prod_{i=1}^{n} R_{i}$. Then, $R$ is endo-Noetherian if and only if $R_{i}$ is endo-Noetherian for each $1 \leq i \leq n$.

Example 2.7 Let $\left(F_{i}\right)_{i \in I}$ be an infinite family of fields and $R=\prod_{i \in I} F_{i}$. Since $R$ is a zerodimensional ring, by [12, Remark 1.20], $R$ is strongly Hopfian. But by Theorem 2.5, $R$ is not an endo-Noetherian ring.

Remark 2.8 Note that if $R$ is endo-Noetherian and $I$ an ideal of $R$, then $R / I$ is not endoNoetherian in general. In fact, let $K$ be a field and $R=K\left[X_{0}, \ldots\right]$, then $R$ is endo-Noetherian. Let $I$ be the ideal of $R$ generated by the elements $X_{0} X_{1}, X_{0}^{2} X_{2}, \cdots$. Then, $R / I$ is not strongly Hopfian, because $\overline{X_{n}} \in a n_{R / I}\left(\overline{X_{0}^{n}}\right) \backslash a n n_{R / I}\left(\overline{X_{0}^{n-1}}\right)$. In particular, $R / I$ is not endoNoetherian.

Let $R$ be a commutative ring and let $(*)$ be the following property: every increasing sequence of the form $\left(I: a_{1}\right) \subseteq\left(I: a_{2}\right) \subseteq \cdots$ is stationary, where $I$ is an ideal of $R$ and $\left(a_{k}\right)_{k}$ a sequence of elements of $R$. Note that if $R$ satisfies the property (*), then $R$ satisfies the accr condition (we say that $R$ satisfies the accr condition if the ascending chain of colon ideals of the form $(I: a) \subseteq\left(I: a^{2}\right) \subseteq\left(I: a^{3}\right) \subseteq \ldots$ is stationary for each $a \in R$ and every ideal $I$ of $R$ ). It is easy to show that if $R$ satisfies the property (*), then $R$ is an endo-Noetherian ring and the converse does not hold in general. It suffices to take an integral domain which is not satisfied accr. Indeed, let $F$ be a field, $\mathbf{X}=\left\{X_{i} \mid i \in \mathbb{N}\right\}$ a set of indeterminates over $F$ and $R=F[\mathbf{X}]$. Let $Y$ be an indeterminate over $R$. Since $R$ is not Noetherian, by [16, Theorem 2], $R \llbracket Y \rrbracket$ does not satisfy accr, in particular $R \llbracket Y \rrbracket$ does not satisfy $(*)$. Note that $R \llbracket Y \rrbracket$ is endo-Noetherian, because it is an integral domain.

Theorem 2.9 The following assertions are equivalent for a commutative ring $R$.

1. $R / I$ is an endo-Noetherian ring for each ideal $I$ of $R$.

2. $R$ satisfies the property $(*)$.

Proof $(1) \Rightarrow(2)$. Let $I$ be an ideal of $R$ and $a_{1}, a_{2}, \ldots \in R$ such that $\left(I: a_{1}\right) \subseteq\left(I: a_{2}\right)$ $\subseteq \cdots$. We show that $\operatorname{ann}_{R / I}\left(\overline{a_{1}}\right) \subseteq \operatorname{ann}_{R / I}\left(\overline{a_{2}}\right) \subseteq \cdots$. Let $\bar{\alpha} \in \operatorname{ann}_{R / I}\left(\overline{a_{k}}\right)$, then $\alpha a_{k} \in I$; so $\alpha a_{k+1} \in I$. Therefore, $\bar{\alpha} \in \operatorname{ann}_{R / I}\left(\overline{a_{k+1}}\right)$.

Now, since $R / I$ is endo-Noetherian, there exists a positive integer $n$ such that $\operatorname{ann}_{R / I}\left(\overline{a_{k}}\right)=\operatorname{ann}_{R / I}\left(\overline{a_{n}}\right)$ for each $k \geq n$. We will prove that $\left(I: a_{k}\right)=\left(I: a_{n}\right)$ for each $k \geq n$. Let $b \in\left(I: a_{k}\right)$. Then, $\bar{b} \overline{a_{k}}=\overline{\overline{0}}$ in $R / I$. Therefore, $\bar{b} \in \operatorname{ann}_{R / I}\left(\overline{a_{k}}\right) \subseteq \operatorname{ann}_{R / I}\left(\overline{a_{n}}\right)$; so $b a_{n} \in I$.

$(2) \Rightarrow(1)$. Let $a_{1}, a_{2}, \ldots \in R$ such that $\operatorname{ann}_{R / I}\left(\overline{a_{1}}\right) \subseteq a_{n n_{R / I}}\left(\overline{a_{2}}\right) \subseteq \cdots$. We show that $\left(I: a_{1}\right) \subseteq\left(I: a_{2}\right) \subseteq \cdots$. Let $b \in\left(I: a_{k}\right)$. Then, $\bar{b} \in \operatorname{ann}_{R / I}\left(\overline{a_{k}}\right) \subseteq \operatorname{ann}_{R / I}\left(\overline{a_{k+1}}\right)$. Thus, $b \in\left(I: a_{k+1}\right)$.

Now, by hypothesis, there exists a positive integer $n$ such that $\left(I: a_{k}\right)=\left(I: a_{n}\right)$ for each $k \geq n$. This implies that $a n n_{R / I}\left(\overline{a_{k}}\right)=a n_{R / I}\left(\overline{a_{n}}\right)$ for each $k \geq n$.

Proposition 2.10 Let $R, T$ be two commutative rings and let $f: R \rightarrow T$ be a ring homomorphism. If $R$ satisfies $(*)$, then $f(R)$ satisfies $(*)$. In particular, $f(R)$ is an endo-Noetherian ring. 
Proof Let $I$ be an ideal of $f(R)$ and $a_{1}, a_{2}, \ldots \in R$ such that $\left(I:_{f(R)} f\left(a_{1}\right)\right) \subseteq\left(I:_{f(R)}\right.$ $\left.f\left(a_{2}\right)\right) \subseteq \cdots$. We show that $\left(f^{-1}(I):_{R} a_{1}\right) \subseteq\left(f^{-1}(I):_{R} a_{2}\right) \subseteq \cdots$. Let $x \in R$ such that $x a_{k} \in f^{-1}(I)$; so $f(x) \in\left(I: f(R) f\left(a_{k}\right)\right)$. Therefore, $f(x) \in\left(I: f(R) f\left(a_{k+1}\right)\right)$, and hence $x a_{k+1} \in f^{-1}(I)$.

Now, since $R$ satisfies $(*)$, there exists a positive integer $n$ such that $\left(f^{-1}(I):_{R} a_{k}\right)=$ $\left(f^{-1}(I):_{R} a_{n}\right)$ for each $k \geq n$. We show that $\left(I:_{f(R)} f\left(a_{k}\right)\right)=\left(I: f(R) f\left(a_{n}\right)\right)$ for each $k \geq n$. Let $y \in f(R)$ such that $y f\left(a_{k}\right) \in I$. There exists $x \in R$ such that $y=f(x)$. Then, $f\left(x a_{k}\right) \in I$. This implies that $x a_{k} \in f^{-1}(I)$. Thus, $x a_{n} \in f^{-1}(I)$. Hence, $y f\left(a_{n}\right) \in I$.

Recall the following definitions: A ring $R$ is called a zero divisor ring (a $Z D$-ring), if the set of zero divisors on the $R$-module $R / I$ is a finite union of prime ideals for each ideal $I$ of $R$. A Laskerian ring is one for which every ideal is a finite intersection of primary ideals, and finally, a ring $R$ is strongly Laskerian if it is Laskerian and for each ideal $I$ of $R$ there exists $k \in \mathbb{N}$ such that $(\sqrt{I})^{k} \subseteq I$. Clearly, every Laskerian ring is a ZD-ring. Heinzer and Ohm [11], proved that a polynomial ring $R[X]$ is a ZD-ring if and only if $R$ is Noetherian, and hence the conditions Noetherian, Strongly Laskerian, Laskerian, and ZD are equivalent in $R[X]$. Similarly, Gilmer and Heinzer [8] proved that a power series ring $R \llbracket X \rrbracket$ is Laskerian if and only if $R$ is Noetherian (but $R$ need not be Noetherian if $R \llbracket X \rrbracket$ is a ZD-ring). We remark that $\mathrm{Lu}[16]$ gave a very simple proof to show that if $R[X]($ resp. $R \llbracket X \rrbracket)$ satisfies accr, then $R$ is Noetherian. Using this result, we prove the following proposition.

Proposition $2.11 R[X]$ (resp., $R \llbracket X \rrbracket)$ satisfies $(*)$ if and only if $R$ is Noetherian.

Proof Since $R[X]$ satisfies $(*), R[X]$ satisfies accr; so by [16, Theorem 2], $R$ is Noetherian. The other implication is obvious.

Remark 2.12 By Proposition 2.11, the conditions (*), Noetherian, Strongly Laskerian, Laskerian, and ZD are equivalent in $R[X]$. This shows that the property (*) does not passe to the polynomial (resp., formal power series) rings. Indeed, we will construct a commutative ring $R$ satisfies the condition (*), but $R$ is not Noetherian. Let $K$ be a field and $V$ an infinitedimensional $K$-vector space. By Example 2.1(2), $R=K(+) M$ is not Noetherian. On the other hand, by [2, Corollary 3.4], every proper ideal of $R$ has the form $I=0(+) N$ for some submodule $N$ of $M$. Since $R / I=(K(+) M) /(0(+) N) \simeq K(+)(M / N)$, by Example 2.1(2), $R / I$ is endo-Noetherian for every ideal $I$ of $R$. Thus by Theorem $2.9, R$ satisfies (*).

In [4], D'Anna and Fontana considered a different type of construction obtained involving a ring $R$ and an ideal $I \subseteq R$ that is denoted by $R \bowtie I$, called amalgamated duplication, and it is defined as the following subring of $R \times R$ :

$$
R \bowtie I=\{(r, r+i) \mid r \in R, i \in I\} .
$$

According to [6], an element of $R$ is called a regular element if it is not a zerodivisor. Also, we say that $I$ is a regular ideal if it contains a regular element of $R$. We end this section by the following result.

Theorem 2.13 Let $R$ be a commutative ring and $I$ a regular ideal of $R$. Then, the following assertions are equivalent:

1. $R$ is endo-Noetherian.

2. $R \times R$ is endo-Noetherian.

3. $R \bowtie I$ is endo-Noetherian. 
Proof $(1) \Rightarrow(2)$. It follows from Theorem 2.5.

$(2) \Rightarrow$ (3). Let $\left(r_{1}, r_{1}+i_{1}\right),\left(r_{2}, r_{2}+i_{2}\right), \ldots \in R \bowtie I$ such that $a n n_{R \bowtie I}\left(\left(r_{1}, r_{1}+\right.\right.$ $\left.\left.i_{1}\right)\right) \subseteq \operatorname{ann}_{R \bowtie I}\left(\left(r_{2}, r_{2}+i_{2}\right)\right) \subseteq \cdots$. We will show that $\operatorname{ann}_{R \times R}\left(\left(r_{k}, r_{k}+i_{k}\right)\right) \subseteq$ $\operatorname{ann}_{R \times R}\left(\left(r_{k+1}, r_{k+1}+i_{k+1}\right)\right)$ for each $k \geq 1$. Let $(a, b) \in a n n_{R \times R}\left(\left(r_{k}, r_{k}+i_{k}\right)\right)$ and let $i$ be a regular element of $I$. We have $(i, i)(a, b)=(i a, i a+i(b-a)) \in R \bowtie I$. Moreover, $(i, i)(a, b)\left(r_{k}, r_{k}+i_{k}\right)=(0,0)$, then $(i, i)(a, b) \in a n n_{R \bowtie I}\left(\left(r_{k+1}, r_{k+1}+i_{k+1}\right)\right)$. Thus, $(a, b) \in \operatorname{ann}_{R \times R}\left(\left(r_{k+1}, r_{k+1}+i_{k+1}\right)\right)$, because $(i, i)$ is regular in $R \times R$. Now, by hypothesis, there exists a positive integer $n$ such that $\operatorname{ann}_{R \times R}\left(\left(r_{k}, r_{k}+i_{k}\right)\right)=\operatorname{ann}_{R \times R}\left(\left(r_{n}, r_{n}+i_{n}\right)\right)$ for each $k \geq n$. This implies that for each $k \geq n,(R \bowtie I) \cap a n n_{R \times R}\left(\left(r_{k}, r_{k}+i_{k}\right)\right)=(R \bowtie$ $I) \bigcap \operatorname{ann}_{R \times R}\left(\left(r_{n}, r_{n}+i_{n}\right)\right)$; so $\operatorname{ann}_{R \bowtie I}\left(\left(r_{k}, r_{k}+i_{k}\right)\right)=a n n_{R \bowtie I}\left(\left(r_{n}, r_{n}+i_{n}\right)\right)$ for each $k \geq n$.

(3) $\Rightarrow(1)$. Note that this implication is always true and does not need the assumption that $I$ contains a regular element.

Let $r_{1}, r_{2}, \ldots \in R$ such that $\operatorname{ann}_{R}\left(r_{1}\right) \subseteq \operatorname{ann}_{R}\left(r_{1}\right) \subseteq \cdots$. We will prove that $\operatorname{ann}_{R \bowtie I}\left(\left(r_{k}, r_{k}\right)\right) \subseteq \operatorname{ann}_{R \bowtie I}\left(\left(r_{k+1}, r_{k+1}\right)\right)$ for each $k \geq 1$. Let $(\alpha, \alpha+i) \in R \bowtie I$ such that $(\alpha, \alpha+i)\left(r_{k}, r_{k}\right)=(0,0)$. Then, $\alpha$ and $\alpha+i$ are in $a n n_{R}\left(r_{k}\right)$; so $(\alpha, \alpha+i) \in$ $a n n_{R \bowtie I}\left(\left(r_{k+1}, r_{k+1}\right)\right)$. Since $R \bowtie I$ is endo-Noetherian, there exists a positive integer $n$ such that $a n n_{R \bowtie I}\left(\left(r_{k}, r_{k}\right)\right)=a n_{R \bowtie I}\left(\left(r_{n}, r_{n}\right)\right)$ for each $k \geq n$. Now, we show that $\operatorname{ann}_{R}\left(r_{k}\right)=a n_{R}\left(r_{n}\right)$ for each $k \geq n$. Let $b \in \operatorname{ann}_{R}\left(r_{k}\right)$. Since $(b, b) \in a n n_{R \bowtie I}\left(\left(r_{k}, r_{k}\right)\right)$, $(b, b)\left(r_{n}, r_{n}\right)=(0,0)$ thus $b r_{n}=0$, and hence $b \in \operatorname{ann}_{R}\left(r_{n}\right)$.

\section{The case of Armendariz and power serieswise Armendariz rings}

Recall that a ring $R$ is called an Armendariz ring if whenever the polynomials $f=\sum_{i=0}^{m} a_{i} X^{i}$ and $g=\sum_{i=0}^{n} b_{i} X^{i} \in R[X]$ satisfy $f g=0$ we have $a_{i} b_{j}=0$ for every $i$ and $j$ [14]. We next give a necessary and sufficient condition for the polynomial ring $R[X]$ to be endo-Noetherian, where $R$ is an Armendariz ring.

Theorem 3.1 Let $R$ be an Armendariz commutative ring. Then, the following statements are equivalent:

1. $R[X]$ is endo-Noetherian.

2. $R$ satisfies the acc on annihilators of finite subset.

3. $R$ satisfies the acc on annihilators of finitely generated ideals of $R$.

Proof (1) $\Rightarrow$ (2). Let $S_{1}, S_{2}, \ldots$ be a sequence of finite subset such that $\operatorname{ann}_{R}\left(S_{1}\right) \subseteq$ $\operatorname{ann}_{R}\left(S_{2}\right) \subseteq \cdots$. Put $S_{i}=\left\{a_{0, i}, a_{1, i}, \ldots, a_{n_{i}, i}\right\}$ for each $i$ and let $P_{i}=\sum_{j=0}^{n_{i}} a_{j, i} X^{j}$. We will show that $\operatorname{ann}_{R[X]}\left(P_{1}\right) \subseteq \operatorname{ann}_{R[X]}\left(P_{2}\right) \subseteq \cdots$. Let $k \in \mathbb{N}^{*}$ and let $f=\sum_{j=0}^{d} b_{j} X^{j}$ such that $f P_{k}=0$. Since $R$ is an Armendariz ring, for each $0 \leq j \leq d$ and $0 \leq l \leq n_{k}$, $b_{j} a_{l, k}=0$. Thus for each $0 \leq j \leq d, b_{j} \in \operatorname{ann}_{R}\left(S_{k}\right)$, which implies that for each $0 \leq j \leq d$, $b_{j} \in \operatorname{ann}_{R}\left(S_{k+1}\right)$; so $b_{j} a_{l, k+1}=0$ for each $0 \leq j \leq d$ and $0 \leq l \leq n_{k+1}$. This implies that $f P_{k+1}=0$ and we conclude that $\operatorname{ann}_{R[X]}\left(P_{1}\right) \subseteq \operatorname{ann}_{R[X]}\left(P_{2}\right) \subseteq \cdots$. Now, since $R[X]$ is endo-Noetherian, there exists a positive integer $n$ such that $\operatorname{ann}_{R[X]}\left(P_{k}\right)=\operatorname{ann}_{R[X]}\left(P_{n}\right)$ for each $k \geq n$. It is easy to show that $\operatorname{ann}_{R}\left(S_{k}\right)=\operatorname{ann}_{R}\left(S_{n}\right)$ for each $k \geq n$. Hence, $R$ satisfies the acc on annihilators of finite subset.

(2) $\Rightarrow$ (1) Let $\left(P_{k}\right)_{k}$ be a sequence of elements of $R[X]$ such that $\operatorname{ann}_{R[X]}\left(P_{1}\right) \subseteq$ $\operatorname{ann}_{R[X]}\left(P_{2}\right) \subseteq \cdots$. For each $i \geq 1$, let $P_{i}=\sum_{j=0}^{n_{i}} a_{j, i} X^{j}$ and put $S_{i}=\left\{a_{0, i}, a_{1, i}, \ldots, a_{n_{i}, i}\right\}$. We will prove that $\operatorname{ann}_{R}\left(S_{k}\right) \subseteq \operatorname{ann}_{R}\left(S_{k+1}\right)$ for each $k \geq 1$. Let $\alpha \in R$ such that $\alpha S_{k}=0$. Then by construction of $S_{k}$, we have $\alpha P_{k}=0$; so $\alpha P_{k+1}=0$ which implies that $\alpha S_{k+1}=0$. 
Since $R$ satisfies the acc on annihilators of finite subset, there exists a positive integer $n$ such that $\operatorname{ann}_{R}\left(S_{k}\right)=\operatorname{ann}_{R}\left(S_{n}\right)$ for each $k \geq n$. Now, let $Q=\sum_{i=0}^{r} q_{i} X^{i} \in R[X]$ such that $Q P_{k}=0$. Then $q_{i} S_{k}=0$ for each $0 \leq i \leq r$; so $q_{i} S_{n}=0$ for each $0 \leq i \leq r$. This implies that $Q P_{n}=0$, and hence $a n_{R[X]}\left(P_{n}\right)=\operatorname{ann}_{R[X]}\left(P_{k}\right)$ for each $k \geq n$.

(2) $\Leftrightarrow$ (3). It follows from the fact that for each finitely generated ideal $I=\left(a_{1}, \ldots, a_{n}\right)$ of $R, \operatorname{ann}_{R}(I)=\operatorname{ann}_{R}(S)=\cap_{k=1}^{n} \operatorname{ann}_{R}\left(a_{k}\right)$, where $S=\left\{a_{k} \mid 1 \leq k \leq n\right\}$.

Let $R$ be a ring. According to [12], $R$ is said power serieswise Armendariz if for each $f=\sum_{i \geq 0} a_{i} X^{i}$ and $g=\sum_{i \geq 0} b_{i} X^{i} \in R \llbracket X \rrbracket$ such that $f g=0$, then $a_{i} b_{j}=0$ for each $i$ and $j$. Next, we characterize when the power series rings over power serieswise Armendariz rings are endo-Noetherian. First, we need to recall some results. Note that those results can be found in [12] or [15].

Notation 3.2 Let $R$ be a commutative ring. We note $\operatorname{ann}_{R}\left(2^{R}\right)=\left\{\operatorname{ann}_{R}(U) \mid U \subseteq R\right\}$, and $\operatorname{ann}_{R \llbracket X \rrbracket}\left(2^{R \llbracket X \rrbracket}\right)=\left\{\operatorname{ann}_{R \llbracket X \rrbracket}(V) \mid V \subseteq R \llbracket X \rrbracket\right\}$. If $f \in R \llbracket X \rrbracket$, we note $C_{f}$ the set of all coefficients of $f$ and for $V \subseteq R \llbracket X \rrbracket$, we note $C_{V}=\cup_{f \in V} C_{f}$.

Remark that $\operatorname{ann}_{R \llbracket X \rrbracket}(V) \cap R=\operatorname{ann}_{R}(V)=a n n_{R}\left(C_{V}\right)$ for $V \subseteq R \llbracket X \rrbracket$. So we can define the application

$$
\begin{array}{cl}
\psi: \operatorname{ann}_{R \llbracket X \rrbracket}\left(2^{R \llbracket X \rrbracket}\right) & \rightarrow \operatorname{ann}_{R}\left(2^{R}\right) \\
I & \mapsto \quad I \cap R
\end{array}
$$

Then, $\psi$ is surjective.

Also, let $U \subseteq R$, then $a n_{R}(U) \llbracket X \rrbracket \subseteq \operatorname{ann}_{R \llbracket X \rrbracket}(U)$. Conversely, let $f=\sum_{i \geq 0} a_{i} X^{i}$ such that $f U=0$, then $a_{i} U=0$ for each $i \in \mathbb{N}$; so $f \in \operatorname{ann}_{R}(U) \llbracket X \rrbracket$. Then, $\operatorname{ann}_{R}(U) \llbracket X \rrbracket=$ $a n n_{R \llbracket X \rrbracket}(U)$. Thus, we can define the application

$$
\begin{array}{ccc}
\phi: \operatorname{ann}_{R}\left(2^{R}\right) & \rightarrow & \operatorname{ann}_{R \llbracket X \rrbracket}\left(2^{R \llbracket X \rrbracket}\right) \\
J & \mapsto & J \llbracket X \rrbracket .
\end{array}
$$

Then, $\phi$ is injective.

Proposition 3.3 [15] Let $R$ be a ring. The following are equivalent:

1. $R$ is power serieswise Armendariz.

2. $\phi$ is surjective.

3. $\psi$ is injective.

Now, we are ready to prove the main result of this section. Note that the proof is inspired by [12, Proposition 2.28].

Theorem 3.4 Let $R$ be a power serieswise Armendariz ring. Then, the following statements are equivalent:

1. $R \llbracket X \rrbracket i$ is endo-Noetherian.

2. $R$ satisfies the acc on annihilators of countably subset.

3. $R$ satisfies the acc on annihilators of countably generated ideals of $R$.

4. For each sequence $\left(f_{k}=\sum_{j \geq 0} a_{k, j} X^{j}\right)_{k}$ of elements of $R \llbracket X \rrbracket$ such that ann $n_{R \llbracket X \rrbracket}\left(f_{1}\right) \subseteq$ ann $_{R \llbracket X \rrbracket}\left(f_{2}\right) \subseteq \cdots$, there exists an $n \in \mathbb{N}$ such that for each $k \geq n, \cap_{j \geq 0} a_{R n}\left(a_{k, j}\right)=$ $\cap_{j \geq 0} \operatorname{ann}_{R}\left(a_{n, j}\right)$. 
Proof (1) $\Rightarrow$ (2). Let $S_{1}, S_{2}, \ldots$ be a sequence of countably subset of $R$ such that ann $_{R}\left(S_{1}\right) \subseteq$ $\operatorname{ann}_{R}\left(S_{2}\right) \subseteq \cdots$. For each $i \in \mathbb{N}$, let $S_{i}=\left\{a_{j, i} \mid j \in \mathbb{N}\right\}$ and put $P_{i}=\sum_{j \geq 0} a_{j, i} X^{j} \in$ $R \llbracket X \rrbracket$. In the same way of the proof of Theorem 3.1, we can conclude that $\operatorname{ann}\left(S_{n}\right)=$ $\operatorname{ann}\left(S_{n+1}\right)=\operatorname{ann}\left(S_{n+2}\right)=\cdots$ for some $n \in \mathbb{N}^{*}$. So $R$ satisfies the acc on annihilators of countably subset.

$(2) \Rightarrow(1)$. Let $\left(P_{k}\right)_{k}$ be a sequence of elements of $R \llbracket X \rrbracket$ such that $\operatorname{ann}_{R \llbracket X \rrbracket}\left(P_{1}\right) \subseteq$ $\operatorname{ann}_{R \llbracket X \rrbracket}\left(P_{2}\right) \subseteq \cdots$. For each $i \geq 1$, let $P_{i}=\sum_{j \geq 0} a_{j, i} X^{j}$ and put $S_{i}=\left\{a_{j, i} \mid j \in \mathbb{N}\right\}$. Now, in the same way of the proof of Theorem 3.1, we can conclude that $\operatorname{ann}_{R \llbracket X \rrbracket}\left(P_{n}\right)=$ $\operatorname{ann}_{R \llbracket X \rrbracket}\left(P_{n+1}\right)=\operatorname{ann}_{R \llbracket X \rrbracket}\left(P_{n+2}\right)=\cdots$ for some $n \in \mathbb{N}^{*}$. Hence, $R \llbracket X \rrbracket$ is an endoNoetherian ring.

(2) $\Leftrightarrow(3)$. Obvious.

(1) $\Leftrightarrow$ (4). By Proposition 3.3, $R$ is power serieswise Armendariz if and only if $\phi$ is surjective if and only if $\psi$ is injective. In that case, $\phi \circ \psi=i d$ and $\psi \circ \phi=i d$. Then for each $f \in R \llbracket X \rrbracket, a n n_{R \llbracket X \rrbracket}(f)=\phi \circ \psi\left(a n n_{R \llbracket X \rrbracket}(f)\right)=\phi\left(a n n_{R}(f)\right)=a n_{R}(f) \llbracket X \rrbracket$. It is easy to prove that for each $f=\sum_{j \geq 0} a_{j} X^{j} \in R \llbracket X \rrbracket, \operatorname{ann}_{R}(f)=\cap_{j \geq 0} a \operatorname{ann}_{R}\left(a_{j}\right)$. Now, $R \llbracket X \rrbracket$ is endo-Noetherian if and only if for each sequence $\left(f_{k}=\sum_{i>0} a_{k, j} X^{j}\right)_{k}$ of elements of $R \llbracket X \rrbracket$ such that $\operatorname{ann}_{R \llbracket X \rrbracket}\left(f_{1}\right) \subseteq \operatorname{ann}_{R \llbracket X \rrbracket}\left(f_{2}\right) \subseteq \cdots$, there exists an $n \in \mathbb{N}$ such that for each $k \geq n, \operatorname{ann}_{R \llbracket X \rrbracket}\left(f_{k}\right)=\operatorname{ann}_{R \llbracket X \rrbracket}\left(f_{n}\right)$ if and only if $\operatorname{ann}_{R}\left(f_{k}\right) \llbracket X \rrbracket=\operatorname{ann}_{R}\left(f_{n}\right) \llbracket X \rrbracket$ if and only if $\cap_{j \geq 0} \operatorname{ann}_{R}\left(a_{k, j}\right)=\cap_{j \geq 0} \operatorname{ann}_{R}\left(a_{n, j}\right)$.

Acknowledgements The authors would like to thank the referee for his/her insightful suggestions towards the improvement of the paper.

\section{References}

1. Anderson, D.D., Dumitrescu, T.: S-Noetherian rings. Commun. Algebra 30, 4407-4416 (2002)

2. Anderson, D.D., Winders, M.: Idealization of a module. Rocky Mt. J. Math. 1, 3-56 (2009)

3. Benhissi, A.: Chain condition on annihilators and strongly Hopfian property in Hurwitz series ring. Algebra Colloq. 21, 635-646 (2014)

4. D’Anna, M., Fontana, M.: An amalgamated duplication of a ring along an ideal, the basic properties. J. Algebra Appl. 6, 443-459 (2007)

5. D’Anna, M., Fontana, M.: An amalgamated duplication of a ring along a multiplicative canonical ideal. Arkiv Mat. 6, 241-252 (2007)

6. Finocchiaro, C.A.: Prüfer-like conditions on an amalgamated algebra along an ideal. Houst. J. Math. 40, 63-79 (2013)

7. Facchinia, A., Nazemian, Z.: Modules with chain conditions up to isomorphism. J. Algebra 453, 578-601 (2016)

8. Gilmer, R., Heinzer, W.: The Laskerian property, power series rings and Noetherian spectra. Proc. Am. Math. Soc. 79, 13-16 (1980)

9. Hamed, A., Gouaid, B., Benhissi, A.: Rings satisfying the strongly Hopfian and $S$-strongly Hopfian properties. Math. Reports (in press)

10. Hamed, A., Hizem, S.: $S$-Noetherian rings of the forms $\mathcal{A}[X]$ and $\mathcal{A}[[X]]$. Commun. Algebra 43, 38483856 (2015)

11. Heinzer, W., Ohm, J.: On the Noetherian-like rings of E. G. Evans. Proc. Am. Math. Soc. 34, 73-74 (1972)

12. Hizem, S.: Formal power series over strongly hopfian rings. Commun. Algebra 39, 279-291 (2011)

13. Hmaimou, A., Kaidi, A., Campos, E.S.: Generalized fitting modules and rings. J. Algebra 308, 199-214 (2007)

14. Kim, N., Lee, Y.: Armendariz rings and reduced rings. J. Algebra 223, 477-488 (2000)

15. Kim, N.K., Lee, K.H., Lee, Y.: Power series rings satisfying a zero divisor property. Commun. Algebra 34, 2205-2218 (2006)

16. Lu, C.P.: Modules and rings satisfying ACCR. Proc. Am. Math. Soc. 117, 5-10 (1993) 
17. Ndiaye, M.A., Gueye, C.T.: On commutative EKFN-ring with ascending chain condition on annihilators. Int. J. Appl. Math. 86, 871-881 (2013)

Publisher's Note Springer Nature remains neutral with regard to jurisdictional claims in published maps and institutional affiliations. 